\title{
Dermatitis de contacto ocupacional por tintas gráficas de curado ultravioleta (UV)
}

\section{Occupational contact dermatitis from ultraviolet (UV) curing graphic}

\author{
Juan P. Russo ${ }^{1 *}$ y Alicia B. Cannavó
}

${ }^{1}$ Servicio de Dermatología, HIGA San Martín La Plata; ${ }^{2}$ Servicio de Dermatología, Hospital de Clínicas San Martín. Buenos Aires, Argentina

\section{Resumen}

La dermatitis alérgica por contacto ocupacional (DACO) es una alteración frecuente en la industria de las artes gráficas. Las tintas de curación con radiación ultravioleta (UV) suelen ser una gran fuente de sensibilización en estos trabajadores. La prueba del parche es una herramienta clave para el tratamiento de la DACO generada por monómeros de metacrilato que componen este tipo de productos.

Palabras clave: Dermatitis por contacto. Tintas gráficas. Metacrilato. Ocupacional.

\section{Abstract}

Occupational contact allergic dermatitis is common disease in the graphic arts industry. UV healing inks are often an important source of sensitization in these workers. Patch test is a key tool for the management of occupational contact allergic dermatitis generated by methacrylate monomers that compose this type of product.

Key words: Contact dermatitis. Graphic inks. Methacrylate. Occupational.

\section{Introducción}

Se calcula que más del $50 \%$ de los trabajadores de las artes gráficas sufren DACO a lo largo de su vida laboral; las formas irritativas son las más comunes y se deben al uso de jabones, detergentes y solventes, entre otros agentes irritantes. En los últimos 50 años, las DACO por tintas de impresión se han relacionado en su mayor parte con compuestos de metacrilatos de curación con radiación UV o barnices de utilización gráfica.
Una característica muy particular de los monómeros de metacrilatos es su bajo peso molecular cuando no están polimerizados o secos. Esto hace posible que este hapteno posea una gran penetración a través del estrato corneal y de ciertos materiales, como ropa de trabajo o guantes de látex. Una vez que se cura o seca pierde esta propiedad de traspasar y producir dermatitis de contacto.

Los metacrilatos son causa de las DACO por adhesivos que usan los odontólogos, mecánicos dentales, icencia CC BY-NC-ND (http://creativecommons.org/licenses/by-nc-nd/4.0/). 


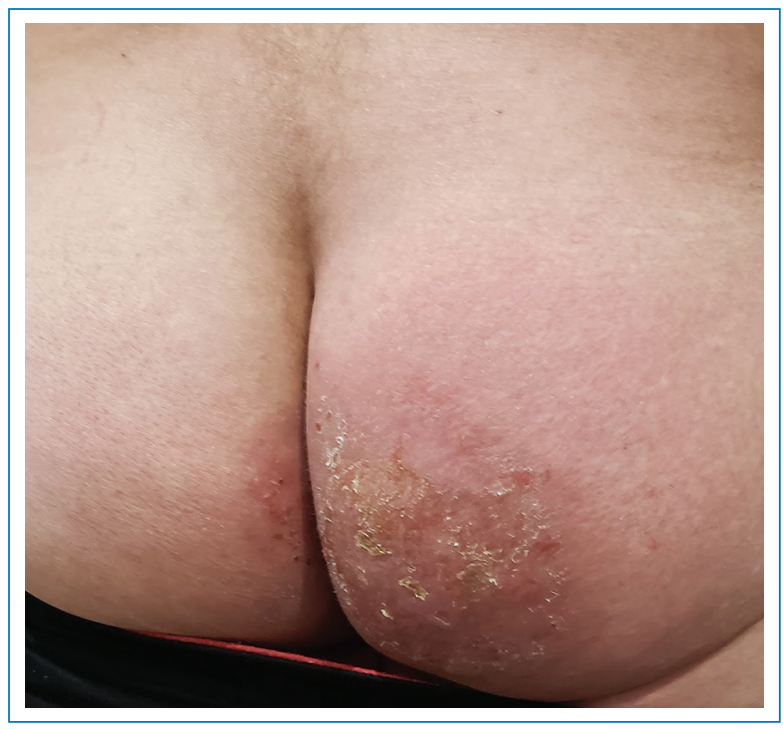

Figura 1. El paciente mostró en la exploración física lesiones de tipo eccema en la región de los glúteos; señaló que las anomalías mejoraban los fines de semana.

traumatólogos e incluso técnicas de colocación de uñas o pestañas postizas, uñas esculpidas, geles ungueales 0 esmaltes semipermanentes. Existen múltiples publicaciones sobre DACO por metacrilato, pero son muy pocos los informes de casos o trabajos sobre DACO por tintes de curación con luz UV.

\section{Presentación del caso}

Paciente del género masculino de 45 años, operario de imprenta desde hace un año sin antecedentes médicos de relevancia. Según refiere, comienza con brotes generalizados de lesiones inflamatorias pruriginosas en distintos lugares del cuerpo de seis meses de evolución. Con anterioridad trabajó en otras imprentas donde tenían diferentes sistemas de impresión convencional, sin aparición de ningún tipo problema relacionado con éstos.

La exploración física revela lesiones eritematosas y escamosas en placas muy pruriginosas en cuello, tronco, axilas y glúteos (Fig. 1 a 3). Se indicó tratamiento con betametasona ( $0.5 \mathrm{mg}$ diarios por vía oral), desloratadina (5 $\mathrm{mg}$ diarios por vía oral), clobetasol (tópico una vez al día) y medidas higiénicas, con lo cual mejoró de modo parcial el cuadro clínico durante dos semanas.

Con posterioridad, al cabo de 15 días, se valoró la posible relación ocupacional del cuadro clínico. El

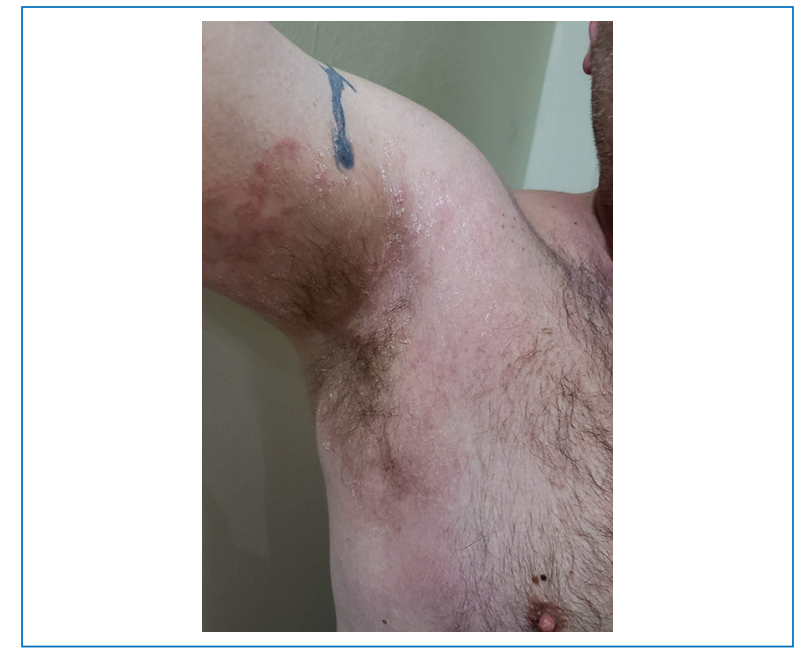

Figura 2. El paciente reveló en la exploración física lesiones de tipo eccema en la región de las axilas.

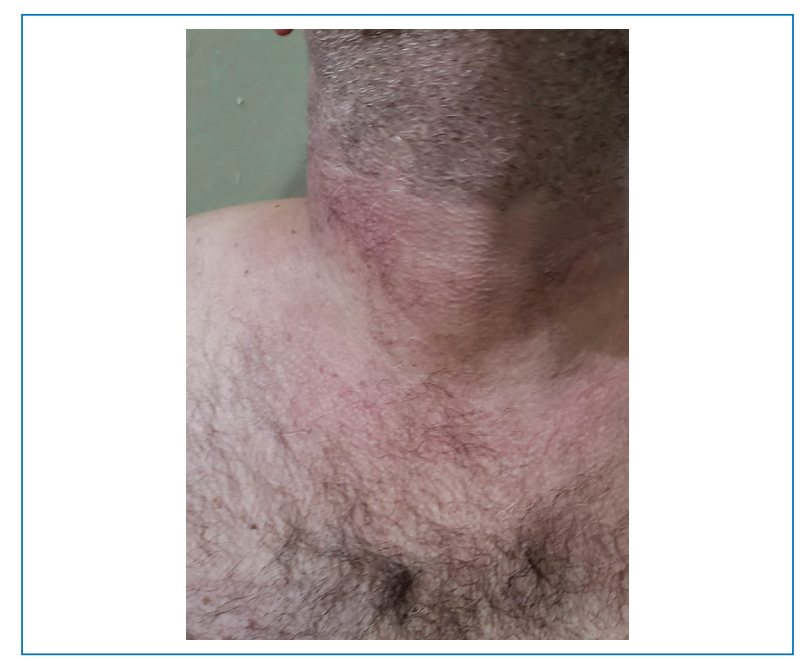

Figura 3. La exploración física identificó en este paciente lesiones de tipo eccema en la región del cuello.

paciente señaló que utiliza guantes, mascarillas y gafas como medidas de protección laboral, pero no suele vestir ropa de operario adecuada, como camisolín o mameluco, ni zapatos de seguridad, sólo ropa de ocasión habitual.

Al consultarlo sobre su actividad laboral específica, llevó una muestra de tinta con la cual tiene contacto y las hojas de seguridad de ésta. Se identifica que la tinta contiene acrilatos de curado UV además de benzofenona como fotoiniciador del producto (Fig. 4 y 5).

El paciente indica que la tinta usada en las máquinas de impresión salpica muchas veces y ensucia otros 


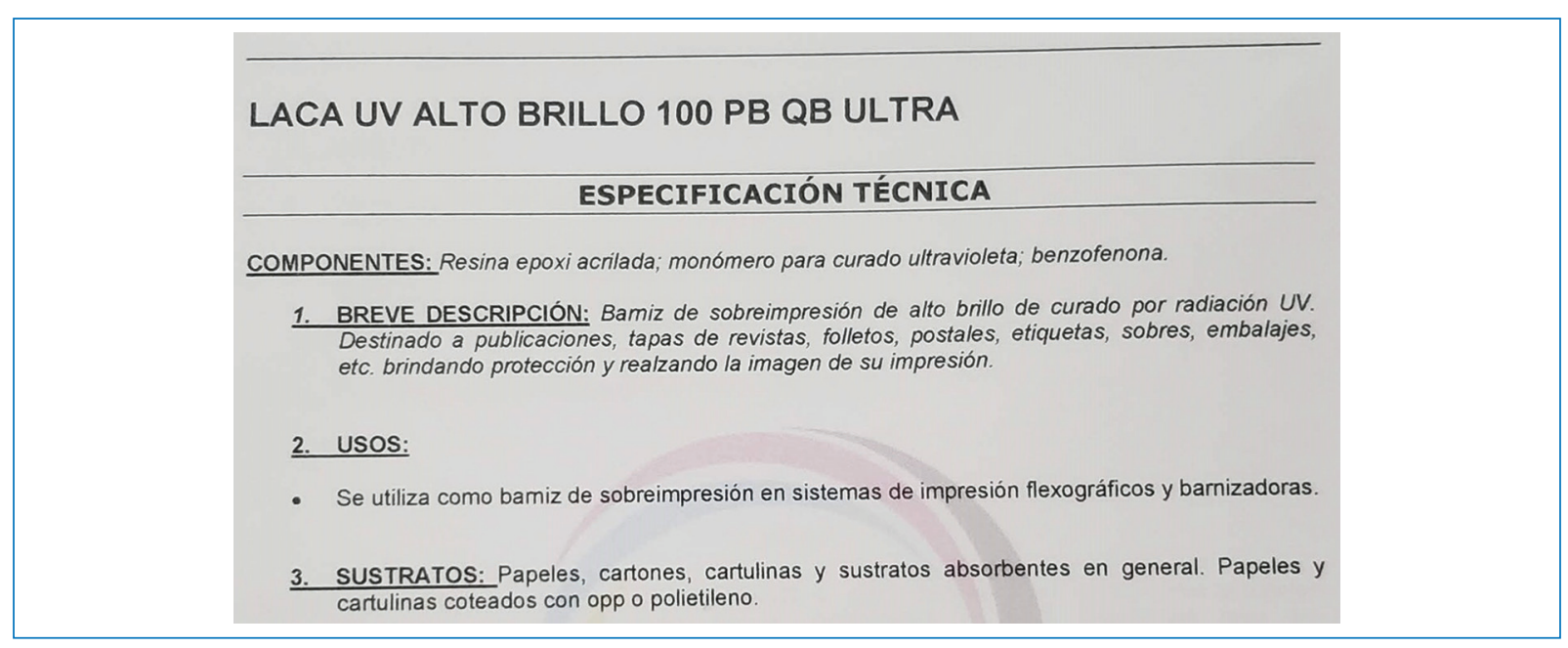

Figura 4. Hoja de seguridad del producto sospechoso (tinta de curación UV) que detalla en su composición la utilización de monómeros acrílicos.

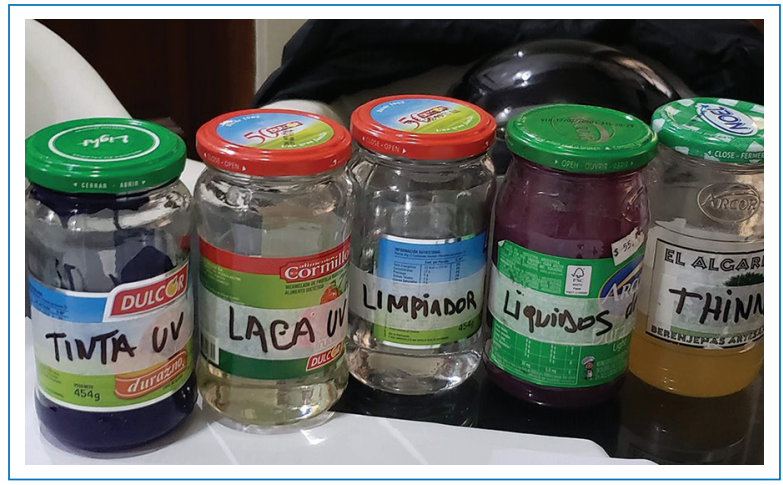

Figura 5. Muestras de los productos que manipula el paciente con la máquina de impresión.

sitios donde trabaja, al igual que sus herramientas e incluso la puede sentir y oler de forma volátil en el medio ambiente laboral. Se realiza la prueba del parche con la Batería de Alergenos Estándar Latinoamericana y la Batería de Resinas de Metacrilato, además de agregar dos alergenos de filtros solares como las benzofenonas 3 y 10 .

Al cabo de las 96 horas de la prueba del parche se observan reacciones positivas a distintos metacrilatos: etilenglicol dimetacrilato (EGDMA) al 2\%: +; 2-hidroxietil metacrilato (2-HEMA) al 1\%: ++; hidroxipropil metacrilato (HPMA) al 2\%: ++; metilmetacrilato (MMA) al 2\%:+; trietilenglicol dimetacrilato (TREGDMA) al $2 \%$ : ++ ; tetrahidrofurfuril metacrilato (THFMA) al 2\%:++; 1,6-hexanediol diacrilato (HDDA) al $0.1 \%$ : +++ (tabla 1 y Fig. 6).

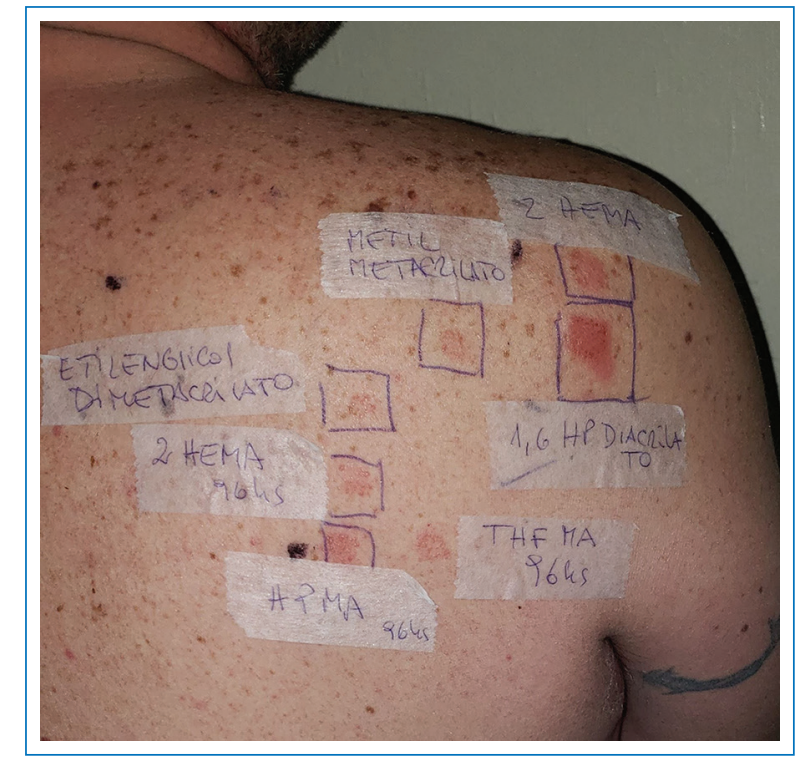

Figura 6. Segunda lectura a las 96 horas de la prueba del parche en la que se observan múltiples sensibilizaciones a los metacrilatos.

Todas las reacciones de la prueba del parche a las 96 horas se interpretan como relevantes y se establece el diagnóstico de DACO por tintes de curación UV que contienen metacrilatos. A continuación se indica cambio de puesto de trabajo y continuar con el tratamiento farmacológico instituido por dos semanas más. Al cabo de un mes sin exposición a los tintes, el paciente se encuentra en remisión completa de su enfermedad alérgica ocupacional. 
Tabla 1. Lectura a las 96 horas tras la prueba del parche de la batería de metacrilatos

\begin{tabular}{|c|c|c|c|}
\hline \multicolumn{4}{|c|}{ Lectura a las 96 horas } \\
\hline 1 & BIS-GMA al $2 \%$, vaselina & (BIS-GMA) & - \\
\hline 2 & $\begin{array}{l}\text { Butanodiol dimetacrilato al } 2 \% \text {, } \\
\text { vaselina }\end{array}$ & (BDMA) & - \\
\hline 3 & $\begin{array}{l}\text { Etilenglicol dimetacrilato al } 2 \% \text {, } \\
\text { vaselina }\end{array}$ & (EDMA) & + \\
\hline 4 & $\begin{array}{l}\text { Hidroxietil metacrilato al 1\%, } \\
\text { vaselina }\end{array}$ & (HEMA) & ++ \\
\hline 5 & $\begin{array}{l}\text { Hidroxipropil metacrilato al } 2 \% \text {, } \\
\text { vaselina }\end{array}$ & (HPMA) & ++ \\
\hline 6 & Metil metacrilato al $2 \%$, vaselina & (MMA) & + \\
\hline 7 & $\begin{array}{l}\text { Trietilenglicol dimetacrilato al } 2 \% \text {, } \\
\text { vaselina }\end{array}$ & (TREGDMA) & ++ \\
\hline 8 & $\begin{array}{l}\text { Uretano dimetacrilato al } 2 \% \text {, } \\
\text { vaselina }\end{array}$ & (UDMA) & - \\
\hline 9 & Etil cianoacrilato al $10 \%$, vaselina & (ECA) & - \\
\hline 10 & $\begin{array}{l}\text { Tetrahidrofurfuril metacrilato al } \\
2 \% \text {, vaselina }\end{array}$ & (THFMA) & ++ \\
\hline 11 & $\begin{array}{l}\text { 2-Hidroxipropil metacrilato al } 2 \% \text {, } \\
\text { vaselina }\end{array}$ & (2-HEMA) & ++ \\
\hline 12 & $\begin{array}{l}1,6 \text { hexanediol diacrilato al } 0.1 \% \text {, } \\
\text { vaselina }\end{array}$ & $(1,6 \mathrm{HDA})$ & +++ \\
\hline 13 & $\begin{array}{l}\text { Uretanodiacrilato aromático al } \\
0.05 \% \text {, vaselina }\end{array}$ & (UDAA) & - \\
\hline 14 & BIS-EMA al $2 \%$, vaselina & (BIS_EMA) & - \\
\hline
\end{tabular}

\section{Discusión}

Las resinas acrílicas son polímeros termoplásticos de ácido acrílico y sus ésteres, extremadamente versátiles, desde elastómeros flexibles y ligeros hasta plásticos termoestables rígidos y duros. La polimerización de los monómeros acrílicos se obtiene por temperatura ambiental, calentamiento o radiación UV'1.

Los acrilatos de curación UV sensibilizan en muchas aplicaciones, entre ellas tintas, lacas, resinas dentales compuestas, moldes para audífonos y cosméticos de uñas ${ }^{2}$.

Según un estudio canadiense, se calcula que alrededor del $65 \%$ de los trabajadores de las imprentas sufre dermatitis por contacto, de las cuales se considera que el $30 \%$ corresponde a DACO por tintes UV curados $^{3}$.

Las tintas fotosensibles UV están compuestas por barnices a los que se le añaden pigmentos. Estos barnices UV tienen tres componentes principales: monómeros acrílicos multifuncionales, oligómeros acrilatos insaturados y un fotoiniciador como la benzofenona. Los pigmentos son sustancias insolubles, resultado del colorante soluble con sales metálicas ${ }^{4}$.

El éster de ácido acrílico y de pentaeritritol (PETA), trimetilol-propano (TMPTA) o hexanodiol diacrilato (HDDA) son los más empleados como acrílicos multifuncionales en tintes UV curables. También pueden contener en su sistema diluyente al prepolímero monoacrilato, como hidroxipropil metacrilato (HPMA) ${ }^{5}$.

En la industria de impresión es frecuente que, además del contacto directo, este tipo de sustancias se volatilice en el medioambiente. Dado que son monómeros haptenos o sustancias de bajo molecular, atraviesan la vestimenta de entramado grueso y guantes de látex con facilidad y provocan dermatitis irritativas y alérgicas por contacto e incluso urticaria por contacto ${ }^{6,7}$. Ésa es la razón por la cual este paciente tenía afectadas zonas vestidas, como axilas y glúteos.

Además, este trabajador de imprenta desarrolló una sensibilización a varios monómeros que componen la batería de metacrilatos, algunos seguramente relacionados con la composición de la tinta, aunque en la hoja de seguridad no se indicaba cuáles eran y otros probablemente eran reacciones cruzadas. Dentro de estas sustancias a destacar figuran el 1,6 hexanodiol diacrilato (HDDA). Éste se considera un sensibilizador moderado a fuerte en tintes UV curables $^{8}$.

Se ha comunicado que el HDDA puede causar eritema similar al multiforme por contacto en trabajadores de imprenta con tintes UV curados $^{9}$. El paciente también tenía sensibilización al tetrahidrofurfuril metilacrilato (THFMA), un acrilato multifuncional muy utilizado en adhesivos médicos, cementos odontológicos y óseos, y también empleado en la industria de impresiones gráficas ${ }^{10}$.

Otro hapteno con probable relevancia en el cuadro clínico del paciente fue el etilenglicol dimetacrilato (EGDMA), un metacrilato multifuncional vinculado con tintes UV curados ${ }^{11}$.

Asimismo, estaba sensibilizado a dos metacrilatos bifuncionales, 2 HEMA y HPMA, ambos también incluidos en tintas UV curadas, sobre todo en la técnica de serigrafía ${ }^{12,13}$. En fecha reciente se publicó un caso de dermatitis ocupacional por tintes UV curados con úlceras y ampollas en antebrazos provocada por un nuevo metacrilato multifuncional Ilamado 2-butilaminocarbonilexietil acrilato $^{14}$. 
Existen informes sobre fotoalergias de contacto al fotoiniciador empleado, casi siempre benzofenonas ${ }^{15}$; en este caso no se consideró la prueba del fotoparche (fotopatch test) por sufrir lesiones en zonas fotoprotegidas, como tronco, axilas y glúteos.

\section{Conclusiones}

La dermatitis por contacto, en sus formas irritativa y alérgica, es frecuente en la industria de las artes gráficas. Este paciente desarrolló una dermatitis alérgica por contacto a los metacrilatos utilizados en los tintes UV curados. Estos monómeros, dado que son haptenos, tienen un alto poder de penetración en la ropa y por ello mostraba afectación en cuello, tronco, axilas y glúteos. Es importante destacar la necesidad de reconocer los agentes causales, así como también realizar la prueba del parche para establecer un correcto diagnóstico y tratamiento.

\section{Agradecimientos}

Los autores expresan su agradecimiento al maestro Luis Conde-Salazar Gómez por su generosidad en transmitirnos su pasión por las dermatitis de contacto.

\section{Responsabilidades éticas}

Protección de personas y animales. Los autores declaran que para esta investigación no se han realizado experimentos en seres humanos ni en animales.

Confidencialidad de los datos. Los autores declaran que han seguido los protocolos de su centro de trabajo sobre la publicación de datos de pacientes.
Derecho a la privacidad y consentimiento informado. Los autores han obtenido el consentimiento informado de los pacientes y/o sujetos referidos en el artículo. Este documento obra en poder del autor de correspondencia.

\section{Bibliografía}

1. Conde-Salazar Gómez L, Romaguera Sagrera C. En: Resinas acrílicas. Dermatitis de contacto de Giménez Camarasa JM. Editorial Aula Médica. 1era edición. 1999. Cap.19.2:261-269.

2. Brooke RC, Beck MH. A new source of allergic contact dermatitis from UV-cured (meth)acrylate adhesive. Contact Dermatitis. 2002:47:179-180.

3. Nethercott JR, Nosal R. Contact dermatitis in printing tradesmen. Contact Dermatitis. 1986;14:280-287.

4. Conde-Salazar Gómez L. En: Dermatitis profesionales en trabajadores de artes gráficas y fotógrafos. Dermatología profesional. Conde-Salazar Gómez, Ancona Alayón A. Ed. Aula Médica, 1era. Ed. Cap. 25:341-359.

5. Bjorkner B, Dahlquist I, Freger S. Allergic contact dermatitis from acrylates in ultraviolet curing inks. Contact Dermatitis. 1980:6:405-409.

6. Nethercott JR. Skin problems associated with multifunctional acrylic monomers in ultraviolet curing inks. British Journal of Dermatology. 1978;98:541.

7. Higgins E, Collins P. Urticarial allergic contact dermatitis caused by UV-cured printing ink. Contact Dermatitis. 2012;66:340-355.

8. Botella-Estrada R, Mora E, De la cuadra J. Hexanediol diacrylate sensitization after accidental occupational exposure. Contact Dermatitis. 1992;26:51

9. Ido T, Kiyohara T, Takahashi H, Yamaguchi $Y$, Tani D. Toxic epidermal necrolysis following allergic contact dermatitis caused by occupational exposure to ultraviolet-cured Inks. Acta Derm Venereol. 2012;92: 313-315.

10. Moffitt DL, Sansom JE Occupational allergic contact dermatitis from tetrahydrofurfuryl acrylate in a medical-device adhesive. Contact Dermatitis. $2001 ; 45: 54$.

11. Goossens A, Coninx D, Rommens K, Verhamme B. Occupational dermatitis in a silk-screen maker. Contact Dermatitis. 1998;39:40-42.

12. Jolanki R, Kanerva L, Estlander T, Tarvainen K. Concomitant sensitization to triglycidyl isocyanurate, diaminodiphenylmethane and 2-hydroxyethyl methacrylate from silk-screen printing coatings in the manufacture of circuit boards. Contact Dermatitis. 1994;30:12-15.

13. Bjorkner B. Contact allergy to 2-hydroxypropyl methacrylate (2-HPMA) in an ultraviolet curable ink. Acta Derm Venereol. 1984:64:264-267.

14. Wahlkvist $\mathrm{H}$, Kaaman AC. Occupational contact allergy to 2-butylaminocarbonyloxyethyl acrylate in UV-curing printing inks. Contact Dermatitis. 2020;1-2.

15. Infante Hernando L, Serra-Baldrich E, Dordal T, Puig Sanz L. Photoallergic contact dermatitis caused by benzophenones in magazine inks. Contact Dermatitis. 2013;69:118-127. 